\title{
Comparison of the Efficacy of Two Different Types of Splint Used in Patients with TemporomandibularJoint Disorders
}

\author{
Kilic Kerem ${ }^{1}$, Eraslan Ravza Kurtulus ${ }^{1}$ and Leblebicioğlu Ikbal*2 \\ ${ }^{1}$ Department of Prosthodontics, Erciyes University, Turkey \\ ${ }^{2}$ Kayseri Nimet Bayraktar Oral and Dental Health Hospital, Turkey
}

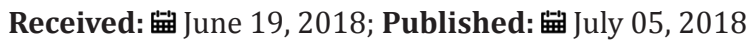

*Corresponding author: Ikbal Leblebicioglu Kurtulus, DDS PhD, ORCID ID: 0000-0001-9122-9852, Kayseri Nimet Bayraktar Oral and Dental Health Hospital, Kayseri/Turkey

\begin{abstract}
Objectives: One of the most common treatment modalities for patients with a diagnosis of reduced disc displacement is occlusal splinting. Stabilization splints (SS) and anterior repositioning splints (ARS) are frequently used in such cases.

Materials and Methods: The effects of these two splint types on patient satisfaction were evaluated in patients with temporomandibular disorder (TMD). 25 healthy people with no pathology of the temporomandibular joint were included as a control group. ARS's were used in 18 patients; all patients used the splints for 2 months. The splint was then converted into a stabilization splint for use for 1 further month. In the other 32 patients, a SS was used for 1 month. All the patients filled out the same temporomandibular questionnaire (TQ) again after the ARS was converted into a SS and had been used for 1 month.

Results: The use of both types of splint significantly affected scores on the TQ $(p<0.05)$. There was a significant difference in questionnaire results among the control group, SS group, and ARS group before treatment $(p<0.05)$. There was no statistically significant difference in questionnaire results among the three groups after treatment $(p>0.05)$.

Conclusion: These results indicate that the use of both ARS and SS significantly increased satisfaction among patients with TMD.

Keywords: Temporomandibular Disorder; Stabilization Splint; Anterior Repositioning Splint

Abbreviations: AD: Alzheimer's Disease; ABETA: Amyloid Beta; BVFTD: Behavioural Variant Frontotemporal Dementia; CMBS: Cerebral Microbleeds; C90RF72: Chromosome 9 Open Reading Frame 72; FTLD: Frontotemporal Lobar Degeneration; HPTAU: Hyperphosphorylated Tau-Protein; INPH: Idiopathic Normal Pressure Hydrocephalus; LBDS: Lewy Body Diseases; MRI: Magnetic Resonance Imaging; PTS: Patients
\end{abstract}

\section{Introduction}

The temporomandibular joint plays an important role in the basic functions of the stomatognathic system. It is a morphologically slipping axis compound joint that makes a hinge and slip movement and varies morphologically among individuals, and between leftand right-sided joints [1,2]. Temporomandibular disorder (TMD) is a clinical condition characterized by pain, emission of a sound (clicking or crepitation), and irregular movements in the jaw joint; it is one of the most difficult-to-treat causes of maxillofacial pain [2].There may be unilateral or bilateral irregularity in the temporomandibular joint $[3,4]$. Certain problems in the temporomandibular joint and neuromuscular system constitute TMD [5,6]. The purpose of treating TMD is to correct etiologic and pathogenic abnormalities, relieve disease symptoms, restore joint mobility, and achieve postural healing [6,7]. TMD is not treated by a single method. Conservative non-surgical treatment options are recommended for initial treatment [5,8]. Commonly used physiotherapy treatment options include an occlusal splint, lowdose laser therapy, physical exercises, and drug therapy to relieve inflammation and pain and stimulate tissue healing [9-12].

One method used to treat dysfunction of the temporomandibular joint and muscles of the chewing system is occlusal splint therapy $[13,14]$. The aim of occlusal splint therapy is to achieve neuromuscular balance in the chewing system and reduce the damage caused by parafunctional habits $[13,15]$. Because this therapy is reversible and noninvasive, its use as an initial treatment is recommended. When the occlusal splint is designed according to the etiology of a particular joint disease, symptoms may be reduced $[11,16,17]$. Occlusal splints commonly used in the treatment of TMD include stabilization splints and anterior repositioning splints [16]. Stabilization splints are usually preferred for patients 
with muscle hyperactivity; thus, they can be used in patients with bruxism [15,16]. Other candidate patients are those with local sensitization or chronic myalgia [10]. In addition, this splint can be used to treat patients with retro discitis, which forms after trauma, to reduce the force on the damaged tissue and confer more effective healing $[13,16]$. Stabilization splints are also used in patients with condylar disc irregularities $[11,16]$.

With use of anterior repositioning splints, highly effective results can be obtained, especially in disc irregularities $[15,18]$. In cases of condylar disc irregularity, stabilization can be achieved with an anterior repositioning splint if the stabilization splint proves ineffective [18,19]. The anterior repositioning splint positions the disc and mandible at the anterior region and ensures a better disc-condyle relationship, thus allowing retrodiscaltissues to adapt. It also allows regeneration of retrodiscal tissues and discrete ligaments andalleviate pain by reducing the load on the joint [18]. In this study, the effects of stabilization and anterior repositioning splints on the healing of patients with TMD were evaluated using a questionnaire survey.

Table 1: Temporomandibular joint questionnaire.
The null hypothesis of this study was;

a) There was not any difference between the questionnaire scores of the groups before and after treatment

b) There was not any difference between the treatment efficiency of anterior repositioning splint and stabilization splint

\section{Material and Method}

In total, 50 patients participated in this study; 32 received a stabilization splint and 18 received an anterior repositioning splint. A control group of 25 individuals without TMJ was also recruited. The selection criteria for the control group were as follows:

a) No temporomandibular joint complaints;

b) Good oral hygiene;

c) No psychological disorders;

d) No dental loss; and

e) No restorations in the mouth and a Class 1 occlusion.

Temporomandibular joint questionnaire

Please mark only one box for each question

1)How would you describe the pain that is generally present in your temporomandibular joint?
a) No pain
b) Very mild
c) Light
d) Mild
e) Severe

2)Do you have any problems with biting into food?
a) No problem
b) Few problems
c) Moderate difficulty
d)I $t$ is extremely hard
e) It is impossible

3) Do you have any problems with chewing food?

a) No problem

b) Few problemsModerate difficulty

c) It's It is extremely hard

d) It is impossible

4) Do you have any problems with sleeping?
a) No problem
b) Few problems
c) Moderate difficulty
d) It is extremely hard
e) I cannot sleep 
5) How often do you have headache?

a) Never

b) Once a week

c) Several times a week

d) Almost every day

e) Always

6)How often do you experience pain in your ear?

a) Never

b) Once a week

c) Several times a week

d) Almost every day

e) Always

7)How often do you feel pain, stiffness, or fatigue in your temporomandibular joint in the mornings?

a) Never

b) Once a week

c) Several times a week

d) Almost every day

e) Always

8)How much does pain caused by your temporomandibular joint interfere with your usual work (can also include housework)?

a) Not at all

b) A little

c) To a moderate degree

d) To a large degree

e) To an extremely large degree

9) When you open your mouth, is any sound emitted from your temporomandibular joint?

a) No

b) Rarely

c) Sometimes

d) Much of the time

e) All the time

10) Do you experience a sensation of decreased mouth opening?

a) No

b) Rarely

c) Sometimes

d) Much of the time

e) I cannot open my mouth at all; it gets locked

Members of the control group completed the questionnaire in Table 1 and the members of the control group has not received any splint therapy.The participants of the other groups were selected from among patients who applied to the Faculty of Dentistry of Erciyes University (Kayseri, Turkey) because of problems in their temporomandibular joint, including the presence of a clicking noise, complaints of pain, bruxism, limited mouth opening, or muscular hypertrophy. Detailed joint examinations were performed by one prosthodontist. The patient groups in our study were constituted using patients included in the RDC (reduction disc displacement)/
TMD Group IIa [19]. To select the patients, a diagnostic algorithm was developed that requires separate examination of the right and left joints [20]. Ethical approval for the study was obtained from the Erciyes University Clinical Research Ethics Committee (Decision No: 2016/4).All participating patients were informed of their inclusion in the study, and all signed consent forms.

A stabilization splint at the temporomandibular joint was randomly applied to 32 patients diagnosed with reduced disc displacement. The splint was used constantly for 1 month except 
during eating.Patients using the stabilization splint filled out the questionnaire twice: once before starting treatment and once after 1 month of using the splint.Upper and lower jaw measurements were obtained using alginate (Tropicalgin; Zhermack, BadiaPolesine, Italy). The jaw models were fabricated using dental stone (Denston 3; Ata Dental Stone Products, Ankara, Turkey). Each patient's centric relation was recorded using silicone bite registration material (Colorbite D; Zhermack). The upper jaw model of each patient was transferred to the articulator (SAM SE Articulator Kit NT ART 616MK, München-Germany) (face-bow transfer). Lateral and protrusive movements were also determined with silicone bite registration material. Then, the cast were connected to a semiadjustable articulator was taken in consideration of the lateral and protrusive movements of the lower jaw model. Upper jaw models were printed with hard Essix plaque (Dispodent, Istanbul, Turkey), and a unique occlusal splint was fabricated for each patient.

Acrylic (Imicryl, Konya, Turkey) was added to the splints for patients undergoing stabilization splinting, and the articulator was closed at the position of centric relation. After the acrylic was polymerized, with lateral and protrusive motions taken into account as well as abrasions, the stabilization splint was fabricated. Then, the splint was inserted into the patient's mouth to ensure that optimal occlusion, and adjustments were performed as needed. Finally, the splint was polished, and the patient was provided with information on how to use it.In the other 18 patients, who had RDD, an anterior repositioning splint was used constantly for 2 months except during eating. The splint was then converted into a stabilization splint and used for a further month.Patients using anterior repositioning splints filled out the questionnaire for the first time before treatment onset. The questionnaire was completed again after the patient had used the splint for 1 month following conversion of the anterior repositioning splint into a stabilization splint.Upper and lower jaw measurements were obtained using alginate (Tropicalgin; Zhermack), and dental stone (Denston 3; Ata Dental Stone Products) was used for jaw models of patients who were scheduled to receive an anterior repositioning splint. Silicone bite registration material (Colorbite D; Zhermack) was used to obtain an impression of the patient's lower jaw with the mouth closed in the teeth to teeth position.

Hard Essix plaque (Dispodent) was applied to the upper jaw of all patients, and occlusal splints were fabricated. The face-bow transfer procedure was applied, and the upper jaw model of each patient was transferred to the articulator (SAM SE Articulator Kit NT ART 616MK, München-Germany). The lower jaw models were transferred to the articulator using bite registration, and the lower jaw was positioned at the front. Transparent cold acryl (Imicryl) was applied on top of the occlusal splint, and the articulator was closedcarefully. After the acryl had hardened, premature contact during lateral and protrusive movements were eliminated. Finally, polish was applied, and the patient was instructed regarding correct use of the splint.The fabrication of the stabilization and anterior repositioning splints, as well as observation and follow-up of patients, were performed by a single dentist in the Prosthodontology Department. Both types of splint were worn for 24 hours, except during eating and tooth brushing. The data obtained from the questionnaires were evaluated statistically.

\section{Statistical Analysis}

The normality of the distribution of the data was evaluated using histograms, q-q graphs, and the Shapiro-Wilk test. Differences in categorical variables between groups were assessed by Pearson chisquare. The Wilcoxon test was used to compare the questionnaire scores before and after treatment. For intergroup comparisons of quantitative variables, one-way analysis of variance (ANOVA) and the Kruskal-Wallis test were used. Multiple comparisons were performed using the Tukey and Dunn-Bonferroni tests. The data analysis was done using $\mathrm{R}$ software (ver. 3.4.0; R Development Core Team, Vienna, Austria). A p-value of $<0.05$ was considered significant.

\section{Results}

a) Table 2 shows the questionnaire data of the stabilization splint, anterior repositioning splint, and control groups by gender. According to the results gender did not have a significant effect $(p>0.05)$.

Table 2: The questionnaire data results of the groups by gender

\begin{tabular}{|c|c|c|c|c|c|}
\hline \multicolumn{2}{|c|}{ Variable } & \multicolumn{2}{c|}{ Groups } & \multirow{2}{*}{ Control } \\
\cline { 3 - 5 } & $\begin{array}{c}\text { anterior } \\
\text { repositioning } \\
\text { splint }\end{array}$ & stabilization splint & \multirow{2}{*}{ P } \\
\hline \multirow{2}{*}{ Gender } & Female & $15(\% 83,3)$ & $26(\% 81,2)$ & $17(\% 68)$ & 0,467 \\
\cline { 2 - 5 } & Male & $3(\% 16,7)$ & $6(\% 18,8)$ & $8(\% 32)$ & \\
\hline
\end{tabular}

b) Table 3 shows the questionnaire data of the study groups

before and after treatment. For all questionnaire items, the pre-

intervention scores were higher than the post-intervention scores (all p < 0.05).

Table 3: The questionnaire data of the groups before and after treatment

\begin{tabular}{|c|c|c|c|}
\hline Variables & \multicolumn{2}{|c|}{ Groups } & \multirow{2}{*}{ P } \\
\hline Questions & Before treatment & After treatment & $<0,001$ \\
\hline Q1 & $3(2-4)$ & $0,5(0-2)$ & $<0,001$ \\
\hline Q2 & $2(1-3)$ & $0(0-1)$ & \\
\hline
\end{tabular}




\begin{tabular}{|c|c|c|c|}
\hline Q3 & $2(1-2)$ & $0(0-1)$ & $<0,001$ \\
\hline Q4 & $0(0-2)$ & $0(0-0)$ & 0,001 \\
\hline Q5 & $1,5(0-2)$ & $1(0-1)$ & $<0,001$ \\
\hline Q6 & $1(0-2)$ & $0(0-0)$ & $<0,001$ \\
\hline Q7 & $2(0-3)$ & $0(0-1)$ & $<0,001$ \\
\hline Q9 & $1(0-2)$ & $0(0-1)$ & $<0,001$ \\
\hline Q10 & $3(1-4)$ & $1(0-3)$ & $<0,001$ \\
\hline
\end{tabular}

c) Table 4 shows the pre- and post- intervention questionnaire scores of the patients fitted with an anterior repositioning splint. According to the results, scores were higher on all items, except Question 4, at pre-intervention ( $\mathrm{p}<$ 0.05).

Table 4: The pre and post-intervention questionnaire scores of the patients fitted with an anterior repositioning splint.

\begin{tabular}{|c|c|c|c|}
\hline \multirow{2}{*}{ Variables } & \multicolumn{2}{|c|}{ Groups } & \multirow{2}{*}{ P } \\
\cline { 2 - 4 } & \multicolumn{2}{|c|}{ Anterior repositioning splint } \\
\hline Questions & Before & After & 0,001 \\
\hline Q1 & $2(0-3,25)$ & $0(0-0,25)$ & 0,001 \\
\hline Q2 & $1,5(1-2,25)$ & $0(0-0)$ & 0,001 \\
\hline Q3 & $2(1-2)$ & $0(0-0,25)$ & 0,180 \\
\hline Q5 & $0(0-0)$ & $0(0-0)$ & 0,009 \\
\hline Q6 & $1(0-2)$ & $0(0-1)$ & 0,026 \\
\hline Q7 & $0(0-01)$ & $0(0-0)$ & 0,005 \\
\hline Q9 & $2(0-3)$ & $0(0-1)$ & 0,01 \\
\hline
\end{tabular}

d) According to the pre- and post- intervention questionnaire scores of the patients fitted with a stabilization splint, a statistically significant difference was observed before and after the treatment $(\mathrm{p}<0.05$, Table 5)

Table 5: The pre and post-intervention questionnaire scores of the patients fitted with a stabilization splint.

\begin{tabular}{|c|c|c|c|}
\hline \multirow{2}{*}{ Variables } & \multicolumn{2}{|c|}{ Groups } & \multirow{2}{*}{ P } \\
\cline { 2 - 4 } & Before & After & $<0,001$ \\
\hline Questions & $3(2,25-4)$ & $1(0-3)$ & $<0,001$ \\
\hline Q1 & $2(0,25-3)$ & $0(0-1,75)$ & $<0,001$ \\
\hline Q3 & $2(0-2,75)$ & $0(0-1)$ & 0,003 \\
\hline Q4 & $0(0-2)$ & $0(0-0)$ & $<0,001$ \\
\hline Q6 & $2(1-3)$ & $1(0-1)$ & $<0,001$ \\
\hline Q7 & $1(0-3)$ & $0(0-1)$ & 0,002 \\
\hline Q9 & $3(0,25-3,75)$ & $1(0-2)$ & 0,001 \\
\hline
\end{tabular}

e) Age did not have a significant effect upon the questionnaire and control groups ( $\mathrm{p}>0.05$, Table 6). data of the stabilization splint, anterior repositioning splint, 
Table 6: The questionnaire data of the groups by age.

\begin{tabular}{|c|c|c|c|c|}
\hline \multirow{2}{*}{ Variable } & \multicolumn{3}{|c|}{ Groups } & \multirow{2}{*}{ P } \\
\cline { 2 - 5 } & $\begin{array}{c}\text { Anterior repositioning } \\
\text { splint }\end{array}$ & Stabilization splint & Control & 0,090 \\
\hline Age & $27,72 \mp 16,30$ & $31,46 \mp 9,25$ & $25,44 \mp 4,22$ & 0 \\
\hline
\end{tabular}

f) Table 7 shows the results of the pre- and post-treatment surveys for the groups. A significant difference was observed before and after treatment in all groups $(\mathrm{p}<0.05)$. The anterior repositioning and stabilization splint groups had higher scores than the control group for questions 1-10. In addition; at question 4, stabilization splint group had a higher value than the anterior repositioning splint and control groups.

Table 7: Results of the pre- and post-treatment surveys for the groups.

\begin{tabular}{|c|c|c|c|c|}
\hline Variables & $\begin{array}{c}\text { Groups } \\
\text { Quterior repositioning } \\
\text { splint }\end{array}$ & Stabilization splint & Control & P \\
\hline Questions & $1,5(0-3)^{\mathrm{a}}$ & $1(0-3)^{\mathrm{a}}$ & $0(0-0)^{\mathrm{b}}$ & $0(0-0)^{\mathrm{b}}$ \\
\hline Q1a-Q1b & $1(0,75-2)^{\mathrm{a}}$ & $1(0-2)^{\mathrm{a}}$ & $0(0-0)^{\mathrm{b}}$ & $<0,001$ \\
\hline Q2a-Q2b & $1(0,75-2)^{\mathrm{a}}$ & $1(0-2)^{\mathrm{a}}$ & $0(0-0)^{\mathrm{b}}$ & 0,001 \\
\hline Q3a-Q3b & $0(0-0)^{\mathrm{b}}$ & $0(0-2)^{\mathrm{a}}$ & $0(0-0)^{\mathrm{b}}$ & $<010$ \\
\hline Q4a-Q4b & $0(0-1)^{\mathrm{a}}$ & $1(0-1)^{\mathrm{a}}$ & $0(0-0)^{\mathrm{b}}$ & $<0,001$ \\
\hline Q5a-Q5b & $0(0-1)^{\mathrm{b}}$ & $1(0-2)^{\mathrm{a}}$ & $0(0-0)^{\mathrm{b}}$ & 0,001 \\
\hline Q6a-Q6b & $1(0-2,25)^{\mathrm{a}}$ & $5(0-3)^{\mathrm{a}}$ & $0(0-0)^{\mathrm{b}}$ & 0,003 \\
\hline Q7q-Q7b & $0(0-1)^{\mathrm{a}}$ & $0(0-1)^{\mathrm{a}}$ & $0(0-0)^{\mathrm{b}}$ & $<(0-0)^{\mathrm{b}}$ \\
\hline Q8a-Q8b & $2(0-3)^{\mathrm{a}}$ & $0(0-2)^{\mathrm{a}}$ & 0,001 \\
\hline Q9a-Q9b & $1(0-2,25)^{\mathrm{a}}$ & $0(0-2)^{\mathrm{a}}$ & 0,001 \\
\hline Q10a-Q10b & & 0,001 \\
\hline
\end{tabular}

*Identical letters in the same row indicate similar scores between groups; different letters represent differences between groups.

\section{Discussion}

In studies on the epidemiology of temporomandibular joint irregularities, symptoms have been reported to be most prevalent in the age range of 20 to 40 years, consistent with the findings of our study [20,21]. In children and adolescents, symptoms are more obvious [23]. In adults older than 60 years, temporomandibular joint symptoms are rarely encountered [24]. TMD is the most common joint disease among younger individuals [15, 25]. Possible reasons for this are the limitation of the disease itself, as well as the higher rates of anxiety and number of stressful situations encountered by younger individuals. The average age of the TMD patients in our study is in line with that in other studies [22,23,25]. Studies of TMD patients have shown female/male ratios of 3.1/1: 9/1, [26,27]; however, there are also studies reporting no significant difference in TMD incidence between the genders [27]. Other studies have reported that the high incidence of TMD in women is related to the greater sensitivity of this population to health challenges, the higher levels of stress hormones seen in women, and the negative effects of female reproductive hormones and oral contraceptives on joints [28-30]. In still other studies, in terms of the gender ratio, TMD was again more common in women and the female/male ratio ranged between $2 / 1$ and $9 / 1[28,31]$.

In the present study, $77.33 \%$ of our randomly selected patients were female and $22.67 \%$ were male. This is consistent with the results of the previous studies. In our study, there was no significant difference between the anterior repositioned splint and the stabilization splint groups in terms of age or gender ( $p>0.05)$.Behr et al. and Tecco et al. applied different types of splint to patients with internal TMD. A positive effect of splinting was more obvious in the anterior repositioning splint group than in the splint stabilization group, although the difference was not significant [32,33]. Similar to the findings of Behr et al. and Tecco et al., we found that the use of both an anterior repositioning splint and a stabilization splint significantly affected scores on the temporomandibular questionnaire used in our study $(p<0.05)[32,33]$. Hence the first null hypothesis of our study was rejected. In the study of Hasegawa et al, splint therapy was applied to 75 patients with TMD [34].

Irrespective of the magnetic resonance (MR) findings, splint therapy outcomes were superior in patients with a significant organic defect, such as unilateral anterior disc displacement or joint effusion [34]. In a study by Devi, three different splint types (anterior repositioning splint, stabilization splint, and soft splint) were applied randomly to 30 discs displacement patients aged from 18 to 55 years [35]. The anterior repositioning splint was considered the conventional treatment option [35]. Whether there was a significant difference between visual analog scale pain score values before and after treatment of three groups, there was not any statistically significant difference between visual analog scale pain score of anterior repositioning splint and stabilization splint groups [35]. The stabilization splint group showed a more obvious improvement at follow-up visits versus the soft splint group [35]. 
For this reason, it is proposed that treatment be initiated with a stabilization splint in patients with reduction disc displacement to achieve more rapid and effective results with minimal side effects [35].

Similar to the findings of Devi et al., in our study; there was not any statistically significant difference between anterior repositioning splint and stabilization splint groups in terms of treatment efficiency ( $p>0.05$ ). Consequently, the second hypothesis of the study was accepted. A study by Santacatterina et al. showed that the effectiveness of anterior repositioning splints, against clicking sounds and pain in the joint region, was significantly higher than that of bite plane among patients with reduction disc displacement [36]. Lundh treated TMD patients with reciprocal clicking symptoms using anterior repositioning splints [37]. The results were compared to those of a control group that received no treatment and a group that received flat occlusal splints [37]. Joint sensitivity was decreased in those using the flat occlusal splint, but there was no change in clicking sounds or muscle sensitivity [37]. Clicking sounds persisted in the control group, and an increase in muscle sensitivity was also observed [37]. During rest and protrusion, there was a decrease in joint pain during chewing with use of anterior repositioning splints [37].

Reciprocal clicking was eliminated and sensitivity to palpation in the joint and muscle region decreased [37]. However, these positive effects persisted only for a short period of time [37]. After 6 weeks of splinting, all symptoms (pain, clicking sound, and tenderness) had returned [37]. Therefore, in patients with TMD accompanied by reciprocal clicking, treatment with anterior repositioning splint yielded successful results [37]. However, because symptoms return when splint use is discontinued, it has been suggested that the mandibular position should be constantly changed [37]. In a study by Anderson et al, orthopedic mandibular repositioning and straight occlusal splint therapy were compared in 20 patients with internal TMD reduction [38]. The mandibular repositioning splint significantly improved the dysfunction and muscle pain associated with TMD, and eliminated reciprocal clicking [38]. Straight occlusal splinting has been shown to not significantly affect the degree of dysfunction in TMD [38]. It has seen in the literature that in the TMJ internal disorders, application of anterior repositioning splint and stabilization splint had positive effects. The results of our study also showed that the use of anterior repositioning splint and stabilization splint had positive effects on internal TMJ disease and there was no statistically significant difference in treatment efficacy between these two splints.

As indicated by Lundh et al., in the treatment of TMJ disorders with anterior repositioning splint, the symptoms may return when the mandible can be brought back to its former position after therapy [37]. As the results of our study did not differ between the treatment efficacy of the two splints, and as noted by Lundh et al. [37], the use of a stabilization splint in TMJ internal disorders may be more appropriate due to possible symptom recurrence of the anterior repositioning splint. The limitations of this study were the small numbers of patients included and the inability to evaluate whether symptoms returned in the long-term after treatment.

\section{Conclusion}

However, within these constraints, it can be stated that the use of both anterior repositioning splints and stabilization splints significantly increased the satisfaction of TMD patients.

\section{Acknowledgement}

We would like to thank Dr Gokmen Zararsiz from the department of Biostatistics at Erciyes University, Kayseri, Turkey for his assistance with the statistics in this study. This study was supported by Project No:TSA-2016-6652 by Erciyes University Scientific Research Projects Coordination Unit.

\section{References}

1. Afroz S, Naritani M, Hosoki H, Takechi K, Okayama Y, et al. (2018) Prevalence of Posterior Disc Displacement of the Temporomandibular Joint in Patients with Temporomandibular Disorders: Systematic Review and Meta-Analyses. J Oral Facial Pain Headache p. 25.

2. Nifosi F, Violato E, Pavan C, Sifari L, Novello G, et al. (2007) Psychopathology and clinical features in an Italian sample of patients with myofascial and temporomandibular joint pain: preliminary data. Int J Psychiatry Med 37(3): 283-300.

3. Fredricson AS, Khodabandehlou F, Weiner CK, Naimi-Akbar A, Adami J, et al. (2017) Are there early signs that predict development of temporomandibular joint disease? J Oral Sci 60(2): 194-200.

4. Jerjes W, Upile T, Abbas S, Kafas P, Vourvachis M, et al. (2008) Muscle disorders and dentition-related aspects in temporomandibular disorders: controversies in the most commonly used treatment modalities. Int Arch Med 30: 1-23.

5. Manfredini D, Poggio CE (2017) Prosthodontic planning in patients with temporomandibular disorders and/or bruxism: A systematic review. J Prosthet Dent 117(5): 606-613.

6. Costa YM, Porporatti AL, Stuginski-Barbosa J, Bonjardim LR, Speciali JG, et al. (2015) Headache Attributed to Masticatory Myofascial Pain: Clinical Features and Management Outcomes. J Oral Facial Pain Headache29(4): 323-330.

7. Suvinen TI, Reade PC, Hanes KR, Kononen M, Kemppainen P (2005) Temporomandibular disorder subtypes according to self-reported physical and psychosocial variables in female patients: a re-evaluation. J Oral Rehabil 32(3): 166-173.

8. Khan MT, Verma SK, Maheshwari S, Zahid SN, Chaudhary PK (2013) Neuromuscular dentistry: Occlusal diseases and posture. J Oral Biol Craniofac Res 3(3): 146-150.

9. Kalamir A, Bonello R, Graham P, Vitiello AL, Pollard H (2012) Intraoral myofascial therapy for chronic myogenous temporomandibular disorder: a randomized controlled trial. J Manipulative Physiol Ther 35(1): 26-37.

10. McNeill C (1997) Management of temporomandibular disorders: concepts and controversies. J Prosthet Dent 77(5): 510-522.

11. Dimitroulis G (1998) Temporomandibular disorders: a clinical update. BMJ 317(7152): 190-194.

12. Mazzetto MO, Carrasco TG, Bidinelo EF, de Andrade Pizzo RC, Mazzetto RG (2007) Low intensity laser application in temporomandibular disorders: a phase I double-blind study. Cranio 25(3): 186-192.

13. Ferreira FM, Cezar Simamoto-Junior P, Soares CJ, Ramos A, FernandesNeto AJ (2017) Effect of Occlusal Splints on the Stress Distribution on the Temporomandibular Joint Disc. Braz Dent J 28(3): 324-329.

14. Algabri RS, Alqutaibi AY (2017) No Evidence Suggests that the Clinical Effectiveness of Conventional Occlusal Splints is Superior to That of Psychosocial Interventions for Myofascial Tempromandibular Disorders Pain. J Evid Based Dent Pract 17(4): 399-401. 
15. Okeson JP (2009) Critical commentary 1: Evaluation of the research diagnostic criteria for temporomandibular disorders for the recognition of an anterior disc displacement with reduction. J Orofac Pain 23(4) 312-315.

16. Al-Ani MZ, Davies SJ, Gray RJ, Sloan P, Glenny AM (2004)Stabilisation splint therapy for temporomandibular pain dysfunction syndrome. Cochrane Database Syst Rev 1: 1-24.

17. Liu MQ, Lei J, Han JH, Yap AU, Fu KY (2017) Metrical analysis of disccondyle relation with different splint treatment positions in patients with TMJ disc displacement. J Appl Oral Sci 25(5): 483-489.

18. Gil-Martinez A, Paris-Alemany A, Lopez-de-Uralde-Villanueva I, La Touche $R$ (2018) Management of pain in patients with temporomandibular disorder (TMD): challenges and solutions. J Pain Res 11: 571-587.

19. Look JO, Schiffman EL, Truelove EL, Ahmad M (2010) Reliability and validity of Axis I of the Research Diagnostic Criteria for Temporomandibular Disorders (RDC/TMD) with proposed revisions. J Oral Rehabil 37(10): 744-759.

20. Celakil T, Muric A, GokcenRoehlig B, Evlioglu G (2017) Management of pain in TMD patients: Bio-oxidative ozone therapy versus occlusal splints. Cranio 24: 1-9.

21. Dworkin SF, LeResche L (1992) Research diagnostic criteria for temporomandibular disorders: review, criteria, examinations and specifications, critique. J CraniomandibDisord 6(4): 301-355.

22. Greene CS (1994) Temporomandibular disorders in the geriatric population. J Prosthet Dent 72(5): 507-509.

23. Mintz SS (1993) Craniomandibular dysfunction in children and adolescents: a review. Cranio 11(3): 224-231.

24. Osterberg T, Carlsson GE, Wedel A, Johansson U (1992) A cross-sectional and longitudinal study of craniomandibular dysfunction in an elderly population. J CraniomandibDisord 6(4): 237-245.

25. de Bont LG, Stegenga B (1993) Pathology of temporomandibular joint internal derangement and osteoarthrosis. Int J Oral Maxillofac Surg 22(2): 71-74.

26. List T, Dworkin SF (1996) Comparing TMD diagnoses and clinical findings at Swedish and US TMD centers using research diagnostic criteria for temporomandibular disorders. J Orofac Pain 10(3): 240-253.

27. Yap AU, Dworkin SF, Chua EK, List T, Tan KB, et al. (2003) Prevalence of temporomandibular disorder subtypes, psychologic distress, and psychosocial dysfunction in Asian patients. J Orofac Pain 17(1): 21-28.
28. Randolph CS, Greene CS, Moretti R, Forbes D, Perry HT (1990) Conservative management of temporomandibular disorders: a posttreatment comparison between patients from a university clinic and from private practice. Am J Orthod Dentofacial Orthop 98(1): 77-82.

29. Shaffer SM, Brismee JM,Sizer PS, Courtney CA(2014) Temporomandibular disorders. Part 2: conservative management. J Man Manip Ther 22(1): 13-23.

30.Warren MP, Fried JL (2001) Temporomandibular disorders and hormones in women. Cells Tissues Organs169(3): 187-192.

31. Phillips JM, Gatchel RJ, Wesley AL, Ellis E (2001) Clinical implications of sex in acute temporomandibular disorders. J Am Dent Assoc 132(1): 49-57.

32. Behr M, Stebner K, Kolbeck C, Faltermeier A, Driemel O, et al. (2007) Outcomes of temporomandibular joint disorder therapy: observations over 13 years. Acta Odontol Scand 65(5): 249-253.

33. Tecco S, Festa F, Salini V, Epifania E, D’Attilio M (2004) Treatment of joint pain and joint noises associated with a recent TMJ internal derangement: a comparison of an anterior repositioning splint, a full-arch maxillary stabilization splint, and an untreated control group. Cranio 22(3): 209219.

34. Hasegawa $\mathrm{Y}$, Kakimoto N, Tomita S, Fujiwara M, Ishikura R, et al. (2017) Clinical study of splint therapeutic efficacy for the relief of temporomandibular joint discomfort. J Craniomaxillofac Surg 45(1): 1772-1777.

35. Devi J, Verma M, Gupta R (2017) Assessment of treatment response to splint therapy and evaluation of TMJ function using joint vibration analysis in patients exhibiting TMJ disc displacement with reduction: A clinical study. Indian J Dent Res 28(1): 33-43.

36. Santacatterina A, Paoli M, Peretta R, Bambace A, Beltrame A (1998) A comparison between horizontal splint and repositioning splint in the treatment of 'disc dislocation with reduction'. Literature meta-analysis. J Oral Rehabil 25(2): 81-88.

37. Lundh H, Westesson PL, Kopp S, Tillstrom B (1985) Anterior repositioning splint in the treatment of temporomandibular joints with reciprocal clicking: comparison with a flat occlusal splint and an untreated control group. Oral Surg Oral Med Oral Pathol 60(2): 131-136.

38. Anderson GC, Schulte JK, Goodkind RJ (1985) Comparative study of two treatment methods for internal derangement of the temporomandibular joint. J Prosthet Dent 53(3): 392-397.
ISSN: 2574-1241

DOI: $10.26717 /$ BJSTR.2018.06.001342

Leblebicioğlu Ikbal. Biomed J Sci \& Tech Res

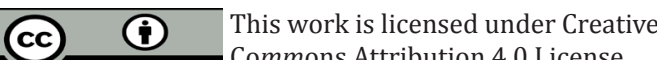

Submission Link: https://biomedres.us/submit-manuscript.php

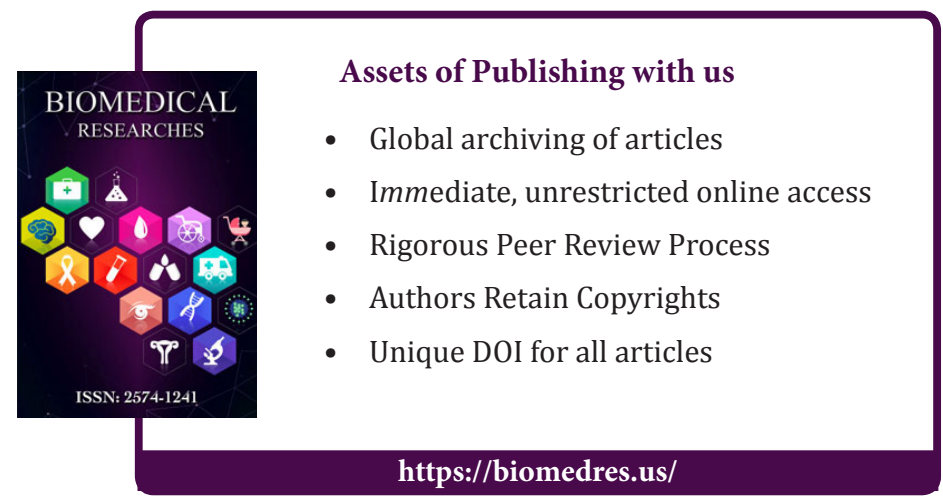

